\title{
Simulating a Team Behaviour of Affective Agents using Robocode
}

\author{
António Rebelo ${ }^{1}$, Fábio Catalão ${ }^{1}$, João Alves ${ }^{1}$, Goreti Marreiros ${ }^{3,4}$, Cesar \\ Analide $^{1,2}$, Paulo Novais ${ }^{1,2}$, José Neves ${ }^{1,2}$ \\ ${ }^{1}$ Universidade do Minho, Campus de Gualtar, 4700 Braga, Portugal, \{pg19827, \\ pg19832,pg20688\}@alunos.uminho.pt \\ ${ }^{2}$ CCTC - Centro de Ciências e Tecnologias da Computação, \{analide, pjon, \\ jneves\}@di.uminho.pt \\ ${ }^{3}$ GECAD - Knowledge Engineering and Decision Support Group, Porto, Portugal, \\ mgt@isep.ipp.pt \\ ${ }^{4}$ Institute of Engineering - Polytechnic of Porto, Porto, Portugal
}

\begin{abstract}
The study of the impact of emotion and affect in decision making processes involved in a working team stands for a multi-disciplinary issue (e.g. with insights from disciplines such as Psychology, Neuroscience, Philosophy and Computer Science). On the one hand, and in order to create such an environment we look at a team of affective agents to play into a battlefield, which present different emotional profiles (e.g. personality and mood).On the other hand, to attain cooperation, a voting mechanism and a decision-making process was implemented, being Robocode used as the simulation environment. Indeed, the results so far obtained are quite satisfying; the agent team performs quite well in the battlefield and undertakes different behaviours depending on the skirmish conditions.
\end{abstract}

\section{Introduction}

Traditionally, emotions and affects have been ignored in classic decision making methods [1]. However, in the last years, researchers of distinct areas (e.g. Psychology, Neuroscience and Philosophy) have begun to explore the role of the affect as a positive influence on human decision-making. Currently, the representation of human emotions in artificial environments is a common issue in Artificial Intelligence.

In 2003, Ortony [2] discussed the main characteristics that an agent must have to be considered believable. There, it was defended that agents should have consistent motivational and behaviours states. In order to ponder this option, it is reinforced that agents need not only a robust model of emotions but 
also have to implement a proper model of personality, which will contribute to give them coherence, consistence and some degree of predictability.

In this work it is proposed the development of dissimilar affective robots and the simulation of their behaviour and performance in a battlefield environment. Undeniably, it will be shaped robots with different emotional profiles and analysed their behaviour, either when act per se or when are part of a team. In order to create robots that may in a consistent way express emotions felt during the course of a battle, and to make the system more similar to human perception, some insights lent from the field of psychology will be considered $[2,17,19]$. As a simulation environment it will be used Robocode, whose objective is to code a robot to beat others in a battlefield [3]. It provides a simple setting, which allows for an easy understanding of robot coding concepts. Robocode is also a very flexible platform, which admits the use of Artificial Intelligence related methodologies and strategies for problem solving in teamwork [4].

This paper comprises in section 2 a brief description of the psychological concepts involved in this work, and in section 3 an overview of Robocode environment and the main movements allowed to robots in a battlefield. Section 4 and 5 presents and discuss our approach to create affective robots in Robocode. Finally, some conclusions are presented in section 6 .

\section{Background}

In this section it will be given a brief description of the main psychological concepts that will be incorporated into the affective.

\section{$2.1 \quad$ Affect}

It is often found in the literature the use of alternative terms such as emotion, affect and mood. Here it is adopted the definition of Forgas [5], that recognises affect as the most generic and used term to refer to mood and emotion. Emotion is normally referred to as an intense experience of short duration (seconds to minutes), with a specific origin, and in general, any person is conscious of the situation. On the other hand, moods have a propensity to be less intensive, longer lasting (hours or even days) and in general remain unacquainted. Moods may be caused by an intense or recurrent emotion, or yet by environmental aspects.

The psychology literature is full of examples on how emotions affect the decision-making process $[6,7,8]$. The frequently changing emotional states of an individual influence their behaviour and their interactions with those around him/her, which in the present context are other group members. For example, the phenomenon of emotional contagion is the tendency to express and feel emotions that are similar to and influenced by others. This phenome- 
non may be analysed as the modal mood of the group, in terms of a particularly salient emotion that one of the group members is feeling [9].

We propose to incorporate emotions into our system using the OCC model of Ortony, Clore and Collins [10], an archetypal that is widely used for emotion simulation of embodied agents [11-15]. In OCC, agent's concerns are divided into goals (i.e. desired states of the world), standards (i.e. ideas on how people should act) and preferences (i.e. likes and dislikes), and distributed across twenty-two properly representable emotion categories or "types". To reduce the complexity in his original model, Ortony proposed a simplified one with 12 (twelve) emotional categories divided into 6 (six) positive (i.e. joy, hope, relief, pride, gratitude, and love) and 6 (six) negative categories (i.e. distress, fear, disappointment, remorse, anger, and hate) [2]. We expect this reduced model to be adequate for our purposes.

It is also possible to find other approaches to infer agents moods, most of them related to the set of agent experimented emotions. Definitely, in this work it will be used the approach proposed by Albert of Mehrabian, that ponders that agent's mood is calculated according to 3 (three) variables, namely Pleasure (P), Arousal (A) and Dominance (D) [16].

\subsection{Personality}

The differences in personality manifest themselves in different ways in all aspects of psychological life (e.g. affect, behaviour, motivation, perception, cognition). Moreover, it may be stated that personality has a key role in the conduct of a particular agent. Agent's individual differences and personalities will interfere and influence aspects of their psychology, such as the way it perceives emotions, feels affection, behaviours, motivations, and cognition [17][18]. Despite the high degree of disagreement around the best way to represent an agent personality, there is some support in favour of the Five Factor Model (FFM) [19], which is the personality model more common in computer applications, and according to it, the individual differences are captured in the form of traits, i.e., Openness, Conscientiousness, Extraversion, Agreeableness and Neuroticism [4]. For these reasons, FFM was chosen as the model to be used in this work.

\section{Robocode environment}

The ROBOCODE is an event driven environment (e.g. robot bumps into a wall and robot hitting another robot). There are 5 (five) main devices which allow for the robot control, i.e., movement (forward, backward), tank-body rotation, gun-rotation, radar-rotation. A battle in Robocode is composed by several rounds; at the beginning is assigned to each robot a fixed level of energy. During the battle, the robots' energy level may decrease and/or increase (e.g. hitting other robot increases the energy level, bump a wall or getting hit 
by a shell decreases its energy level). Once the energy level gets to zero, the robot is simply dismissed; the same happens if other robot hits its peers. To consider a round finished, only one robot may remain in the battlefield.

\section{Affective team}

\subsection{Robots Affective model}

The emotional system is built on two important factors, specifically the emotions and the mood of the robot. These are two distinct components in terms of intensity and duration. The mood of a robot is of low intensity and long duration, while emotions have high intensity but are specific to a particular event, being therefore brief or transitory. The agent personality is paramount to establish its initial mood. In Figure 1, one may see the general approach pursue for the creation of the affective model, in which the environment stands for the framework in which our robot will be immersed, i.e., the physical environment and the other robots that are in the same somatic setting.

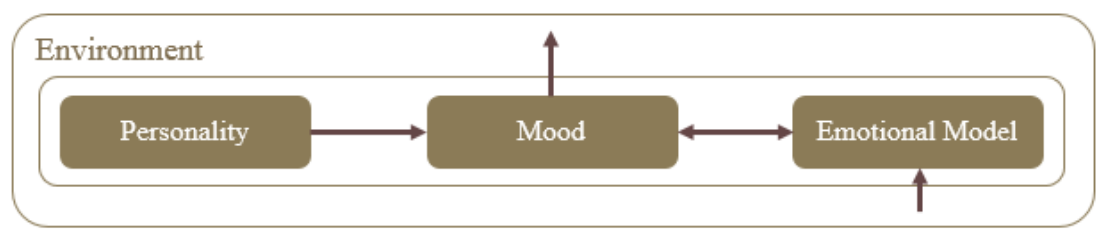

Figure 1 - The affective model

\subsubsection{Modelling Emotions}

Each emotion has an intensity value; however each robot feels emotions with a distinctive strength, which is contingent to several factors. The intensity of the emotion is given in terms of the equation [18]:

Intensity $_{\text {emotion }}=\frac{\sqrt{(P)^{2}+(A)^{2}+(D)^{2}}}{\sqrt{3}}$

These emotions are triggered through their own actions and also through interactions with other robots or even events. As it was mentioned before, the emotions considered in this work are identified in the revised version of the OCC model, i.e., joy, hope, relief, pride, gratitude, love, distress, fear, disappointment, remorse, anger, and hate [2]. Over time, the intensity of emotions decreases or decays, leading to a state of irrelevance; emotion decay is obtained by counting the number of rounds passed since the emotion was felt. The emotion intensity in a given robot is obtained by using the rule [18]:

$I_{\text {emotion felt }(r)}=I * e^{-r} * n(2)$

i.e., the intensity of an emotion felt by a robot varies according to the number of counted rounds $(r)$ since the emotion was triggered, the Intensity of emotion $(I)$ and the value of neuroticism $(n)$ of the robot in question. 


\subsubsection{Modelling Mood}

Emotions change the current mood depending on its intensity and the personality of a robot. The mood of a robot is modelled based on the work of Mehrabian, in terms of Pleasure $(P)$, Arousal $(A)$ and Dominance $(D)$, which forms the $P A D$ space [11]. The $P$ dimension is related to the emotional state of the robot, being positive or negative; the $A$ scale denotes its level of physical and mental activity; the $D$ one indicates its feeling of being in control. Mehrabian defined 8 (eight) types of mood based on $P A D$ values, which were adopted to represent the mood of robots. Mehrabian also established a relationship between the OCEAN model of 5 (five) dimensions with the threedimensional $P A D$ space. Through this relationship it is possible to set the mood of the original robots according to their personality, i.e., the $O C E A N=$ $(O, C, E, A, N)$ model is used to obtain the robots personality values. Therefore, the initial mood of a robot may be calculated as follows [17]:

$$
\begin{aligned}
& P=(O, C, E, A, N), \text { where } O, C, E, A, N \in[-1,1] \\
& \text { Mood }=(P, A, D), \text { where } P, A, D \in[-1,1] \\
& P=0.59 \times A+0.19 \times N+0.21 \times E \\
& A=-0.57 \times N+0.30 \times A+0.15 \times O \\
& D=0.60 \times E-0.32 \times A+0.25 \times O
\end{aligned}
$$

Once the initial mood of a robot is computed, it is necessary to define how it is going to evolve. In one`s case the mood swings are due to the occurrence of events and the actions performed by a robot in combat; the emotions generated may be positive or negative, and follow the pattern presented in the OCC model. Over time, and verifying the absence of emotions, mood stabilizes at its initial values. In the present setting it was decided to use an adaptation of the simplified version of the OCC Model .The emotions Love and Hate have been removed due to their non-application in the context of this work.

\subsection{Esteem}

Due to the conduct of the robots on the ground and the events triggered by them, a robot goes through diverse emotional states, which may be positive, offering a good practice, or negative, resulting in discomfort. Having this in mind, it becomes obvious that a robot will have a good indebtedness with respect to a counterpart that causes good practices and little regard for one that only produces bad experiences.

On the other hand each robot team has a value of esteem for their equals in combat. This assessment is central, once it affects many of the actions of the robots. As an example, let us look at the selection of the next enemy to target. If one of the opponents has a very low esteem, it entertains a high probability of being chosen to be targeted by the voting robot, i.e., the values of esteem have a significant weight in the decision making process and in voting. 


\subsection{Decision, votes and leader}

Each robot has its own personality and mood. In this way, they have different desires and goals. In order to achieve some kind of cooperation, it was necessary to create a leader and a decision mechanism able to focus on two key aspects of the battle: the next target of the opposing teams and the movement that should be adopted.

The leader has the responsibility of holding a referendum and responding to it by publishing the results. The leader should ask for the votes of the other members of the team, even of those that have been lethally wounded, which is the case when too many rounds have passed since the last referendum, or if the target of the team has been slaughtered. Upon the death of the team leader, the element with more energy will announce itself as the new leader and resume the voting mechanism. The entire team votes, giving a preference value to each of the antagonists to defeat and a preference value to each of the possible engagements to use. When the robot evaluates the scores of its enemies, it takes into account the following variables, i.e., its mood, the esteem for that opponent, the opponent's remaining energy and the distance to it.

During the analysis of the votes, the leader may disregard some opinions or give added weight to others. In order to accomplish this practice, the leader takes into account the esteem of the teammate who voted. If the teammate is having a good performance in combat, then the level of appreciation of the leader is high, and so the leader values its opinion. Otherwise, if the teammate is failing too many shots and hitting its own teammates, then the leader has a low level of esteem to it and its opinion is devalued against that of others. When the results of the voting process are treated, the team leader may either accept them and pronounce the results or, if the results are going in a different direction of its own interpretation of the situation on the ground, the team leader may proceed in order to reach its purposes. The probability of the group decision being manipulated by the leader is directly proportional to its value of thoughtfulness and inversely proportional to its value of agreeableness.

\subsection{Movements}

In 2009, Nakagawa work look at a reformation of the motion method to control affective nuances in robots [20]. In order to revise diverse types of motions without changing their meanings, this method uses three parameters: velocity and extension of motion and a basic posture. Following Nakagawa idea, the mood of the robots along the battle is shaped. Robots, when subjected to successive negative emotions tend to do more unwise decisions and to reduce teamwork. When robots are subjected to successive positive emotions they tended to be more relaxed and to increase teamwork.

The robots developed in this project may implement one of the following types of engagement: defensive, opportunistic and tactical. The defensive ro- 
bot has as its main goal to keep itself at a secure distance from its opponents. The opportunist robot changes its self-confident attitude when is struck by a bullet or when someone crashes with it. This is a robot that times its attacks based on the level of security it feels. If it does not take damage in the last rounds, it feels confident and takes the opportunity to get closer to its enemies, improving its chances of striking. However, if an enemy manages to decrease its energy, the opportunistic robot retreats and starts to avoid its nemeses, hoping that its interest on it will fall and may look at another robot. A tactical robot is an entity that performs its actions based on a defined line of behaviour. This robot plans its navigation before the encounter, and executes it disregarding any outside interference. It relies on tactic and perception of the battlefield as it keeps itself near the borderlines of the conflict.

\section{Simulation Analysis}

According to the simulation results, a set of conclusions may be made based on the robots behavior. It can be said that emotions influenced the outcome of the simulations. The emotions shaped the mood of the robots along the battle, which in turn shaped the actions and votes consummated. Successive negative emotions felt by the robots make them more likely to do unwise decisions, bringing down teamwork, which is a typical sign of emotion instability. On the other hand, robots feeling consecutive positive emotions proved to be more relaxed and to cooperate as a team. Simulations have shown that personality played an important role as well, as robots with confident and cool personality would tolerate negative situations and stick to the plan, while others with a more neurotic personality would panic and stray away from the plan. This is consistent with the literature [21], as emotions have different intensities and durations depending on the individual personality. Based on the environment in which the simulation takes place, these types of behavior are in concordance with real life situations. Teams are able to act accordingly to the robots emotions and personality.

\section{Conclusions and future work}

In this work it were presented robots capable of feeling emotions and act accordingly, namely changing its behaviour. It stands for an approach to create an affective team of robots to be used in a battlefield; it was used the $P A D$ mood space, which is able to support OCEAN and OCC models. Each robot of the team has its own personality and mood. In order to achieve cooperation, a voting mechanism and a decision-making process was implemented. The results are quite satisfying as the team nurses a very good performance on the battlefield and assumes diverse conducts that are contingent on the battle conditions. As future work, it is intended to create other types of move- 
ment that a team may endorse, as well as to develop a more multifaceted system to choose the governance.

\section{Acknowledgements}

This work is funded by national funds through the FCT - Fundação para a Ciência e a Tecnologia (Portuguese Foundation for Science and Technology) within project PEst-OE/EEI/UI0752/2011.

\section{References}

1. Marreiros G., Ramos C, Neves J., "Emotion and Group Decision Making in Artificial Intelligence”. Cognitive, Emotive and Ethical Aspects of Decision-Making in Humans and in AI - Vol IV. ISBN 1-894613-86-4, pp. 41-46, 2005.

2. Ortony A., "On making believable emotional agents believable”, In R. P. Traple, P. (Ed.), Emotions in humans and artefacts, Cambridge: MIT Press, 2003.

3. Hartness K., "Robocode: using games to teach artificial intelligence”, J. Comput. Sci. Coll. 19, 4 (April 2004), 287-291.

4. Howard P., Howard J.,'The BIG FIVE Quickstart: An introduction to the five factor model of personality for human resource professionals”. Charlotte, NC: Center for Applied Cognitive Studies, 2004.

5. Forgas J., "Mood and judgment: The affect infusion model (AIM)". Psychological Bulletin, 117, pp. 39-66, 1995.

6. Loewenstein G. and Lerner, J. S., "The role of affect in decision making”, in Handbook of Affective Sciences. Oxford University Press, 2003.

7. Schwarz, N. "Emotion, cognition, and decision making". Cognition and Emotion, 14(4), pp. 433-440, 2000.

8. Barsade S. "The Ripple Effect: Emotional Contagion and Its Influence on Group Behavior”. Administrative Science Quarterly, 47, pp. 644-675, 2002.

9. Neumann R. and Strack F. "Mood contagion: The automatic transfer of mood between persons”. Journal of Personality and Social Psychology, 79 pp 211-223, 2000.

10. Ortony A., Clore GL. and Collins A., "The cognitive structure of emotions", Cambridge: Cambridge University Press,1988.

11. Gratch J. and Marsella S. Evaluating a computational model of emotion. Journal of Autonomous Agents and Multiagent Systems, 11(1), pp. 23-43, 2006

12. Mourão, D. and Paiva A "EToy: Building an affective physical interface”. 2nd Workshop on Attitude, Personality and Emotions in User-Adapted Interaction, 2001.

13. MeiYii, L., Dias J., Aylett R., Paiva A. "Improving Adaptiveness in Autonomous Characters”. Proc IVA 2008 LNAI 5208 pp348-355 Springer

14. Bída M.,Brom C. "Towards a platform for the education in emotion modeling based on virtual environments." 3rd Workshop on Emotion and Computing, pp. 45 - 52, 2008.

15. Marreiros G., Santos R., Ramos C. and Neves J. Context-aware emotion-based model for group decision making. IEEE Intelligent Systems magazine, 25(2), 31-39. 2010

16. R. Ryckman, “Theories of Personality”, Thomson/Wadsworth, 2004.

17. Mehrabian, Analysis of the Big-Five Personality Factors in Terms of the PAD Temperament Model.: Australian Journal of Psychology, 46, 1996

18. Santos R, Marreiros G, Ramos C, Neves J, Bulas-Cruz J, "Personality, Emotion, and Mood in Agent-Based Group Decision Making”, IEEE Intelligent Systems, 26(6), pp. 58-66, 2011.

19. McCrae, R.R. and John, P.O. An introduction to the five-factor model and its applications, Journal of Personality 60, pp.175-215, 1992. 
20. Nakagawa, K.; Shinozawa, K.; Ishiguro, H.; Akimoto, T.; Hagita, N.; , "Motion modification method to control affective nuances for robots," Int. Conf. Intelligent Robots and Systems, pp.5003-5008, 2009

21. Haifang L.; Haipeng H.; JunJie C., "A new layered model of affect," Int. Asia Conf. on Informatics in Control, Automation and Robotics (CAR), 2010, pp.261-264 